\title{
TIEMPO REAL Y TIEMPOS GRAMATICALES: PRESENTE E IMPERFECTO DE INDICATIVO
}

\author{
JOANNA WILK-RACIĘSKA \\ (Universidad de Silesia)
}

\begin{abstract}
The present work deals with the polyfuncionality of two tenses of Spanish indicativo: the presente and the imperfecto, and with the hierarchy of these functions as well.

Tenses aren't an isolated grammatical category, there are rather expressions of relations among different linguistic and extralinguistic dimensions. Thus, the given meaning of a grammatical tense is associated with a function which this tense realizes in a specific relation.

This text seeks to establish an appropriate classification of these functions. It means, that the author's aim is to isolate the prototypical function of each of these tenses and to define reasons and possibilities of its peripherial uses.

Although an expression's meaning and even its grammatical organisation are dependent on context, general knowledge and experience, an important part of each cognitive item is constructed in accordance with stable linguistic patterns. Accordingly, the work tries to discover underlying linguistic patterns (treated here as a prototypical lunction) of analysed constructions and to describe the validity of these patterns for the peripherial use of lexical terms.

Theoretical instruments applied are: the semantic theory known as semantic grammar or semantic syntaxe (St. Karolak, 1991) and the cognitive grammar carried out within the perspective stated by R. Langacker. For the verification of some universal properties and uses of analysed forms appropriate Polish constructions were used.
\end{abstract}

El tiempo pasa, corre, e incluso huye, pero también puede pararse cuando menos lo quercmos y necesitamos; a veces podemos tenerlo, pero, por lo general, nos falta, por lo que es muy importante economizarlo. Unos dicen que el tiempo 
consuela a todos, y otros - como Marco Aurelio- que 'es como un río que arrastra rápidamente todo lo que nace'.

Inventando todas estas metáforas intentamos familiarizarnos con el tiempo real, una dimensión relativa y caprichosa que hasta ahora no hemos conseguido dominar ni con la lísica ni aún con la gramática que nos sirve para expresar nuestra relación con el tiempo.

Aunque en la gramática existe la noción de tiempo como una categoría gramatical del verbo, es muy importante no olvidar que la relación entre el tiempo ontológico y el tiempo gramatical no es unívoca. El hecho de tratar tiempos gramaticales como exponentes del tiempo real ha provocado y sigue provocando muchas discrepancias teóricas. Mientras tanto existen lenguas que se las arreglan perfectamente sin tiempos gramaticales, aunque nadie diría que las naciones que las usan viven sin tiempo real. Basta con mencionar el chino clásico, el hébreo, el idioma khmer o malayo que no conocen esta categoría gramatical y expresan las relaciones temporales sirviéndose de adverbios y expresiones temporales o simplemente del contexto. Otras lenguas, como las semíticas o el Yoruba marcan la dilerencia entre el aspecto perfectivo y el imperfectivo. Por otra parte, las gramáticas de las lenguas románicas (para no extendernos demasiado) suelen mencionar la existencia de un montón de tiempos gramaticales, aunque buena parte de cstos tiempos ha caido en desuso (como, por ejemplo, el pretérito anterior español, la mayoría de formas del subjuntivo francés, etc), y los gramáticos no pueden alcanzar un acuerdo común respecto a la naturaleza de los demás. De todos modos, uno de los hechos irrefutables es que tantos tiempos gramaticales no pueden corresponder a los tiempos reales, ya que vivimos exclusivamente en el PRESENTE, recordamos el PASADO y sólo podemos planear el FUTURO, de lo que resulta otro hecho evidente, a saber, que, con total responsabilidad, sólo podemos hablar de la relación tiempo pasado / tiempo no pasado o sea de una oposición perfectividad / imperfectividad, de la que parece haber surgido una buena parte de las oposiciones marcadas mediante los tiempos gramaticales.

En cste estudio queremos demostrar la polifuncionalidad de dos liempos de indicativo: el presente y el imperfecto, y presentar la jerarquía de estas funciones. Nos ocuparemos, pues, de apartar la función prototípica (básica) de cada una de las formas gramaticales estudiadas, así como de definir tanto las razones como las posibilidades de sus usos periféricos. Teniendo en cuenta el hecho de que la gramática cognitiva defiende que muchas características de la forma lingǘstica ni son arbitrarias ni tampoco son siempre predecibles a partir del significado, sino que están motivadas, suponemos que las funciones prototípicas se pueden explicar mediante factores puramente lingüísticos (semánticos) mientras que la explicación de los usos periféricos de las formas gramaticales mencionadas requiere criterios experienciales. 
Basaremos nuestra propuesta en la sintaxis semántica' que nos permitirá explicar cl aporte puramente semántico del uso de los dos tiempos gramaticales. También nos será útil la visión langackeriana del cognitivismo, la cual nos aportará una claves para entender la relación entre el lenguaje, la cognición y el mundo. Considerando que la gramática cognitiva es un medio que nos abre caminos a la investigación más allá de la lengua, nos permitiremos proponer una solución cognitiva para esta investigación. Sin embargo, lo fundamental en este trabajo será buscar explicaciones semánticas y descubrir la sistematicidad con la que los factores semánticos se manifiestan en todos los usos de una forma dada.

Ahora sólo falta añadir que, siguiendo el principio del carácter universal de los conceptos, nos permitiremos también servirnos -en algunos casos- de la lengua polaca para corroborar nuestras tesis.

En la sintaxis semántica la proposición lógica (predicado+sus argumentos) no tiene carácter temporal, puesto que su predicado constituyente no incluye en su estructura la noción de tiempo. Sin embargo, los predicados de todo tipo incluyen necesariamente la noción de aspecto. El aspecto es, pues, una unidad inherente, consustancial a los predicados y -en el nivel conceptual- el aspecto propio a un predicado no puede cambiar ${ }^{2}$.

Las proposiciones denotan situaciones virtuales, es decir, cada proposición tiene como referentes un conjunto abierto de situaciones posibles. Para afirmar la existencia de una siluación es indispensable localizar en el eje temporal el aspecto de su concepto constitutivo. Para hacerlo nos servimos de coordinantes temporales identificables para el interlocutor. Esto significa que el predicado temporal es un predicado relacional, de orden superior, que abre dos posiciones: una para una proposición comunicada $(\mathrm{PC})$ y otra para una proposición referencial (PR).

\footnotetext{
1 Véase: Bogacki, K \&.Karolak, St. (1991); una aplicación a la lengua española en: Wilk-Racięka, J. (1995).

${ }^{2}$ La granática con basc semántica postula la existencia de dos valores aspectuales básicos: el valor de continuidad y el valor de momentaneidad. Por el primero se entiende una continuidad no delimitada o un conjunto abicrto de momentos contiguos. Los representantes superficiales de estos conceptos cominuos son los radicales de los verbos llamados tradicionalmente de estado y de actividad, como, por ejemplo: ana-ar, quer-er, comprend-(er) o mir-(ar). Por otra parte, los conceptos momentáneos son los representados por explot-(ar), da-(r), perd-(er), gan-(ar) u otros que representen un evento. momontánco. Sin embargo, es importante subrayar que lo expuesto concierne las estructuras predicativas simples, mientras que en las estructuras predicativas compuestas uno u otro aspecto puede dominar sobre el aspecto de la noción constituyente. (crf. St.Karolak (1997) Así, pues, en la proposición: El pobre perro está nuriéndose de hambre - el concepto momentáneo de mor-(ir) llamado por Bello desinente, .,porque luego que uno /.../muere, deja de /.../ norir» (Bello \$625) está dominado por el aspecto continuativo.
} 
En otras palabras, la proposición comunicada es sólo una parte de la estructura temporal la que por sí misma es compleja. Por eso, las desinencias de tiempos gramaticales no son suficientes para localizar la situación denotada en el eje temporal; para hacerlo necesitamos algo más, esto es otra proposición (PR) cuyo único objetivo es determinar el tiempo de la PC. Esta proposición referencial se realiza, por 10 general, mediante complementos circinstanciales de tiempo, adverbios y expresiones temporales. Comparemos:

a) (?) Se fue a Madrid. vs b) Se fue a Madrid ayer / el año pasado/ a las 7 de la mañanct.

y:

c) He ido a Madrid muchas veces vs d) Voy a Madrid

Como vemos, la proposición a) no es autónoma sin complemento de tiempo o contexto alguno, mientras que las proposiciones c) y d) lo son perfectamente. Según la gramática con base semántica esto significa que en el caso de algunos tiempos verbales, la situación del habla puede también desempeñar el papel de la PR. Aquí conviene subrayar que la gramática con base semántica introduce una diferenciación muy rigurosa entre la situación del habla, llamada también origen o punto de referencia y una proposición referencial. Así, pues de acuerdo con la gramática semántica en nuestro estudio seguiremos considerando el origen o punto de referencia como una unidad extralingüística identificada con el momento de habla. Por no estar entroncado con el sistema lingüístico, el origen sólo sirve para establecer una perspectiva narrativa del enunciado pero, por sí mismo, no puede participar en la creación de una unidad puramente lingüística. Por otra parte, nada impide que el punto de referencia desempeñe el papel de la proposición referencial. Sin embargo tal situación sólo es posible en el caso de que el momento de habla y de la situación denotada por la PC sean simultáneas.

Nos parece asimismo importante añadir que -aunque según Langacker (1999:661) la interpretación de los enunciados fuera del contexto es algo que sólo los linguistas pueden hacer, ya que para la mayoría de la gente es un ejercicio artificial - en nuestro estudio, todos los análisis se realizarán, en la medida de lo posible, sobre los ejemplos de las proposiciones autónomas fuera del contexto, puesto que el análisis de los fenomenos lingüísticos básicos como la definición de las funciones primarias de los tiempos gramaticales, efectuado sobre las proposiciones de tipo elemental ${ }^{3}$, es decir autónomas, queda mucho más claro y evidente.

\footnotetext{
${ }^{3}$ Véanse: Bogacki, K \&.Karolak, St. (1991).
} 
Explicando el uso de los tiempos de indicativo las gramáticas españolas distinguen por lo menos cinco funciones del presente:
Actual:
(1) Leo este libro (ahora mismo)
Habitual:
(2) Me levanto a las siete (por costumbre, no ahora).
Histórico: (3) Colón descubre América en el año 1492 (por:'descubrió')
Futuro: (4) El domingo vamos de excursión (por:'iremos')
De mandato: (5) Sales a la calle y me compras el periódico (sustituye al imperativo)

Sin embargo las funciones de tres de estos enunciados, privados de las explicaciones entre paréntesis, ya no parecen tan claras:

\section{Leo este libro.}

Me levanto a las siete.

Sales a la calle y me compras el periódico.

Es evidente que Leo este libro puede denotar una acción actual, pero nada impide que denote también una acción habitual (como 'leer este libro es mi pasión'). De igual modo Me levanto a las siete puede denotar una acción habitual, pero también expresar sorpresa del autor del enunciado que no suele madrugar, mientras que Sales a la calle y me compras el periódico visto sin contexto puede igualmente parecer un fragmento no autónomo (desde el punto de vista sintácticocomunicativo o convencional, si adaptamos la terminología cognitiva) de una narración. Por otro lado, las funciones de los enunciados: Colón descubre América en el año 1492 y El domingo vamos de excursión son muy fáciles de descifrar aunque no llevasen ningún contexto. En el primer caso es ante todo el complemento circunstancial del tiempo que localiza la situación denotada precisamente en el año 1492, pero nuestros conocimientos del mundo tampoco permitirían situarla en el presente (comparemos: Colón descubre América provoca la misma pregunta que Colón descubrió América sin complemento temporal, es decir: ¿Cúando?). En el segundo caso es la economía de la lengua la que no nos permite localizar la situación denotada en el presente (si hoy es domingo no hace falta repetirlo, el enunciado no se volverá más informativo) ${ }^{4}$.

De lo dicho hasta ahora parece resultar que el 'presente' ontológico equivale a la 'situación del discurso'. Es verdad. Cada acto de enunciación sucede en un contexto espacio-temporal cuyo centro es el hablante. Hablando en términos generales, cada acto de enunciación se centra en torno al aquí-y-ahora del hablante. Es precisamente por esta razón que las funciones de las proposiciones en presente gramatical como (1), (2) y (5) analizadas sin complementos temporales o, por lo

\footnotetext{
${ }^{4}$ Para más información sobre este problema véanse también J. Wilk-Racięska (1997), (1998).
} 
menos, un contexto cualquiera no parecen diferenciarse entre sí de un modo evidente. Tales proposiciones nos remiten directamente a la situación de la enunciación que desempeña el papel de la proposición referencial. Enunciadas en el presente real, estas proposiciones no necesitan un 'localizador' puramente lingüístico y en consecuencia la posición para la proposición referencial queda bloqueada. Esto significa que la tarea del locutor, una tarea evidente y perfectamente convencionalizada, es relacionar la PC con el momento de habla. De ello resulta que la función primaria, prototípica de las proposiciones en presente gramatical es denotar las situaciones actuales, es decir simultáneas con la situación de la enunciación:

Leo este libro.// Escribo las cartas.// Voy a la montaña.// Hablas demasiado.// Sales a la calle.

Alora bien, si las proposiciones referenciales no son necesarias $i$ qué función desempeñan los complementos, adverbios y expresiones temporales en las proposiciones de este tipo?

ia) Alora mismo leo este libro vs 1b) Leo este libro siempre que tengo un poco de tiempo

Visto que las proposiciones en presente actual no necesitan localizadores temporales lingüísticos y que las proposiciones (1a) y (1b) no comunican exactamente lo mismo podemos declarar ${ }^{5}$ que:

a) Las proposiciones realizadas como complementos circunstanciales de tiempo en (1a) y (1b) no desempeñan el papel de proposiciones refcrenciales, ya que en tal caso atribuimos este papel a la situación del discurso, sino que sirven para subrayar la continuidad;

b) las diferencias entre (1a) y (1b) se deben a la oposición: referencial / potencial: la proposición (1a) que ya por sí misma denota una situación real, advierte la cxistencia de esta situación en el momento de habla, es decir esta proposición tiene un referente concreto, hecho que simplemente se subraya mediante el uso del complemento temporal actual. Mientras tanto, el complemento de tipo omnitemporal empleado en la proposición (1b) sirve para demostrar el carácter virtual de la proposición (1b). Hablando en términos generales, la proposición de tipo (1b) no afirma la existencia de una situación real, actual, sino que denota un conjunto abierto de situaciones, es decir las situaciones sobre las cuales la proposición ha sido,

\footnotetext{
'Para más información sobre este problema, especialmente en francés, inglés y búlgaro véanse St. Karolak (1997).
} 
es o puede ser verdadera. Comparemos también: Un políglota es una persona versada en varias lenguas;

c) las únicas construcciones nocionales sobre las cuales pueden construirse las proposiciones $\mathrm{cn}$ el presente gramatical son las construcciones imperfectivas, es decir con el aspecto de continuidad dominante. Esta suposición es lógica y, por lo general no requiere ninguna corroboración. Sólo basta con presentar algunos ejemplos. El predicado de ROBAR se basa en cl aspecto momentáneo, ya que el término sólo puede ser verdadero sobre una acción realizada en un momento casi no perceptible de realidad o, por lo menos, físicamente difícil de definir. Por eso es muy difícil de aceptar una proposición :(?)Ahora mismo roban tu coche (vs Ahora mismo leo este libro), mientras que la proposición: Ahora mismo están robando tu coche significa simplememte: AHORA MISMO ESTÁN HACIENDO ALGO PARA QUE TU COCHE SEA ROBADO que revela una construcción conceptual compleja, donde cl aspecto momentáneo del término analizado está dominado por el aspecto continuativo. Así, pues vista en esta óptica, la proposición (?)Ahora mismo roban tu coche es poco acceptable porque a) la presencia del complemento temporal actual en el enunciado impone al. interlocutor que establezca una correspondencia íntima - la concomitancia - entre la situación denotada por la proposición comunicada y la situación del habla, hecho que no es físicamente posible, vista la momentaneidad de la acción denotada. Por otra parte, la única interpretación posible que se da a esta proposición $\mathrm{s}$ i n complemento actual es la de denotar un conjunto abierto de actos repetidos, pues la de continuidad impuesta a toda la construcción por la imposibilidad de establecer la concomitancia entre la situación denotada por la proposición comunicada y la situación del habla. Comparemos: Todos los domingos / cada año / siempre roban tu coche.

Por el momento podemos concluir que los usos llamados 'habitual' y 'omnitemporal' o 'gnóstico' del presente gramatical son usos secundarios respecto al. uso actual que parece ser básico, es decir prototípico.

Pasemos ahora al. presente llamado histórico y al. presente por futuro. Como ya se ha dicho más arriba, las funciones de los enunciados: Colón descubre América en el año 1492 y El domingo vamos de excursión son muy fáciles de descifrar aunque no llevasen ningún contexto. En el primer caso es, pues, el complemento circunstancial de tiempo, el que localiza la situación denotada precisamente en el año 1492. De este modo ambas posiciones abiertas por el predicado temporal están saiuradas, siendo el complemento circunstancial de tiempo una realización superficial de la PR. El uso del presente en la PC no se contrapone a la localización

"La diferencia cntre los dos últimos ya no es objeto de este estudio. 
de la situación denotada en el pasado real. Al. contrario, las proposiciones de este tipo corroboran perfectamente el hecho de que la estructura de la PC no lleva codilicado el tiempo (teniendo codificado el aspecto) y que el único localizador de esta proposición en el eje temporal puede ser la proposición referencial.

¿De dónde viene, pues, el presente histórico? Este tiempo gramatical nos muestra de un modo muy claro la interacción entre la ESTRUCTURA y el USO de la lengua. Es comunmente sabido que sirve para describir de un modo más vivo y eficaz la situación denotada por la PC. La pregunta es ¿Cómo explicar esta función? Asi pues, de lo dicho hasta ahora resulta que es un hecho irrefutable que tal uso sólo es posible gracias a la estructura semántica de las proposiciones temporales (es decir, el hecho que el predicado temporal es dyádico), así como de la función primordial del presente gramatical que consiste en denotar acciones actuales simultáneas con el momento de habla. Ahora basta solamente con añadir que el mismo mecanismo funciona en las proposiciones con el presente de mandato y el presente por futuro.

Sin cmbargo es lógico que las funciones no centrales de las formas gramaticales sean -en la mayoría de casos- debidas a un conjunto de factores tanto lingüísticos como extralingüísticos. Por este motivo, una vez explicada la función primaria del presente y la razón básica de sus usos periféricos, nos parece que otros factores que intervienen en la elección de esta forma para sus usos no prototípicos pueden explicarse perfectamente mediante el funcionamiento de 'schematized interactive frames' sugeridos por R.Langacker (1999:653). Según afirma Langacker, estos cuadros interactivos esquematizados refieren de un modo esquemático al. hablante y al. oyente, al. enunciado, a las facetas relevantes del contexto y a tales factores como intentos de los interlocutores y a lo que cada uno de los interlocutores sabe de los conocimientos previos e intentos del otro. El inventario de cuadros convencionalizados es bastante largo y asequible para los hablantes, que los activan en discursos actuales. No obstante, en nuestra opinión,cada uno de estos cuadros esquematizados debe tener su propia organización interna, su propia lógica. Si bien los cuadros convencionalizados no se conciben dentro de la lingüística cognitiva como estructuras proposicionales, lo cierto es que, en cualquier caso, deben tener propiedades lingüísticas que sienten las bases para su creación. Así, pues, la base para estos cuadros convencionalizados debe ser siempre la lengua o, más precisamente, las reglas semántico- sintácticas internas que la gobiernan. Combinados con la experiencia y / o con otros factores nombrados por Langacker nos permiten crear una nueva unidad, un nuevo valor.

Para respaldar la idea de que las reglas lingüísticas sientan las bases para la creación de los cuadros convencionalizados pasamos a continuación a analizar las funciones del imperfecto de indicativo. 
Las gramáticas explican la función principal del imperfecto de indicativo utilizando una expresión pintoresca : 'el presente del pasado'. Sin embargo teniendo en cuenta que una acción presente es una acción simultánea con el momento de habla y que hay una diferencia muy importante entre "momento de habla " $o$ "punto de referencia" (fenómeno extralingüístico) y 'la proposición referencial' (fenómeno puramente lingüístico) - la definición 'el presente del pasado' parece contradictoria, o por lo menos poco precisa.

Arriba hemos dicho que, según la gramática con base semántica, en el caso del presente de indicativo, el punto de referencia desempeña el papel de la proposición referencial. Recordemos que el predicado temporal abre dos posiciones: una para la proposición que designa la situación y otra para la proposición que sitúa la primera en el cje temporal. En su forma básica la proposición referencial es una proposición circunstancial de tiempo, en forma secundaria son modificadores temporales que representan proposiciones circunstanciales de tiempo en forma condensada. Visto lo dicho, en el caso de una proposición que designa una acción pasada, la simultaneidad del momento de habla con la situación designada no es posible. Desde este punto de vista, el momento de habla es sólo una perspectiva (extralingüística) de la que se designan las acciones y la única información que éste nos proporciona es que estas acciones ya no tienen lugar. No en balde dijo Amiel: 'el tiempo no es sino un espacio entre nuestros recuerdos' La proposición referencial, es decir la que sitúe, de modo más preciso, la proposición comunicada en el eje del tiempo debe ser simultánea con la proposición comunicada y debe buscarse necesariamente en la lengua (si no se refiere la PC a una época que se supone conocida, que también puede presentarse en forma de una PR). Asimismo esta simultaneidad entre ambas proposiciones (la que queremos comunicar y la referencial):

1) depende del aspecto de sus conceptos constituyentes y

2)puede ser total o parcial.

Una relación de simultaneidad total sólo es posible cuando el aspecto de la proposición comunicada así como el de la proposición referencial es perfectivo (en este caso la proposición referencial puede ser expresada por un complemento circunstancial temporal momentáneo como por ej.: a las dos de la tarde, en este momento):

'Pedro entró en el jardín justamente en el momento cuando disparó el fusil'

'A las dos (en punto)/ en este momento llamaron a la puerta'

No obstante el imperfecto, que expresa conceptos del aspecto imperfectivo (básico o dominante) y no acepta los modificadores temporales momentáneos: 
*En el momento cuando disparó el fusil, Pedro leyó un libro / Pedro estuvó en el jardín

sólo puede entrar en relación de simultaneidad parcial ${ }^{7}$. Comparemos:

Cuando entraste, llovía; (Gili y Gaya)

Mientras unos lloraban, otros reían (Gili y Gaya)

En el segundo ejemplo tenemos que ver con dos lapsos de tiempo no delimitados, mientras que en el primero, la proposición en el indefinido delimita una coincidencia entre un lapso de tiempo momentáneo y una parte del lapso de diempo no delimitado de un modo concreto, de la proposición en el imperfecto. Por otra parte, el ejemplo:

\section{'Durante el examen Manolo contestaba sin reflexionar'}

tambićn corrobora la idea aquí presentada, más el carácter imperfectivo de los conceptos en el imperfecto. Es lógico que en este caso se trate del aspecto imperlectivo dominante y el imperfecto significa aquí las acciones repetidas. El uso del modificador temporal durante permite alargar el tiempo de la situación denolada. Comparemos:

* 'Durante el examen Manolo contestó sin reflexionar' vs 'En el examen Manolo contestó sin reflexionar.

En conclusión, los conceptos del aspecto básico imperfectivo se expresan de modo natural mediante el imperfecto, entrando en la relación de simultaneidad parcial con las proposiciones construidas también a base de los conceptos imperfectivos, o sea con las proposiciones construidas a base de los conceptos básicamente perlectivos (?Pedro entró cuando María estuvo en casa ys Pedro entró cuando Maria estaba en casa). En una pareja de este tipo una de las proposiciones es la proposición comunicada y la otra la proposición referencial.

Las reglas de la gramática semántica - presentadas aquí de un modo muy breve por necesidad-corroboran el hecho comúnmente conocido de que la función prototípica del imperfecto es expresar la continuidad en el pasado. De aquí su nombre 'presente del pasado'. Claro está que, desde un punto de vista extralinguístico, es decir tomando en consideración el momento de habla, la

\footnotetext{
${ }^{7}$ La relación parcial atañe también las parejas: proposición comunicada en indefinido - complemento circunstancial que designa un lapso de tiempo como: ayer, el año pasado. Sin embargo este problema, así como el problema del aspecto perfectivo excede los límites de nuestro estudio.
} 
diferencia consiste en la oposición simultaneidad / anterioridad a este momento, respectivamente. Sin embargo la gramática con base semántica explica ante todo la diferencia puramente lingüística entre estas dos formas gramaticales, que se reduce a la diferencia entre las proposiciones referenciales. La función de la proposición relerencial de una proposición comunicada en el presente la desempeña el mismo punto de relerencia, mientras que, en el caso de una proposición expresada en imperlecto, el punto de referencia sólo puede funcionar como perspectiva del discurso y su proposición referencial debe expresarse también en el texto, en uno de los liempos gramaticales del pasado. La proposición en el imperfecto, puede también, por sí misma, funcionar como proposición referencial para otras proposiciones.

No obstante, la función de expresar la continuidad en el pasado, aunque es prototípica, no es la única entre las funciones del imperfecto. Gili y Gaya enumera las siguientes:

I. 'Al. amanecer salió el ejercito, atravesó la montaña, y poco después cstablccía contacto con el enemigo'-donde, según el autor, se anula el carácter imperfecto de la forma gramatical;

2. 'Lc dio un dolor tan fuerte que se moría' - imperfecto de conatu, donde se sobrepone el aspecto a la significación temporal, que se emplea el imperfecto para acciones que no se han producido todavía;

3. 'Qucría pedirle un favor' - imperfecto de cortesía, cuyo uso en lugar del presente lo explica el aspecto de acción verbal inacabada. «El sentido temporal es presente, aunque enunciamos nuestro deseo en imperlecto, como algo iniciado cuya consumación o perfección hacemos depender de la voluntad de la persona a quien nos dirigimos» (Gili y Gaya, 1979:161);

4. 'Si tuvicra dinero compraba esta casa', en lugar de „compraría' - en la apódosis de las condicinales se emplea con significado futuro. Es un uso raro en la lengua literaria, pero en el habla corriente, posible también fuera de las condicionales;

5. 'Yo cra la princesa, tú eras la reina' - los niños se valen con frecuencia del imperfecto de indicativo en lugar del futuro hipotético para resaltar el carácter de ficción.

En nucstra opinión, todos los cinco empleos periféricos del imperfecto enumerados por Gili y Gaya ( y otros autores españoles de un modo más o menos aproximado) son función de la continuidad más otros factores linguíísticos y cxtralinguísticos, sicndo la continuidad el factor más importante. Comparemos cl primer cjemplo, donde, según Gili y Gaya 'se anula el carácter imperfecto de la forma gramatical'. Este uso del imperfecto es frecuente no sólo en español, sino 
también en otras lenguas románicas como por ejemplo el francés, con el objetivo de poner a la vista la situación denotada, "dramatizarla, "alargando la acción:

A midi, le ministre montait dans sa voiture et partait pour sa maison de campagne. Un moment plus tard, des motards masqués qui l'avait suivi lobligeaient à s'arrêter sur le côté de la route. Ils étaient armés. (Marie Reichler-Béguelin)

Tal procedimiento sólo es posible gracias al. aspecto imperfectivo de los conceptos que forman base de las proposiciones en imperfecto. No obstante la corroboración más fuerte de este factor la puede ser aquí la traducción polaca de las proposiciones ciladas, tanto las españolas como las francesas. El idioma polaco no dispone de un abánico de tiempos pasados: sólo posee uno. Sin embargo, los verbos polacos disponen de dos formas verbales: una perfectiva y otra imperfectiva. Así pues, las partes de las proposiciones citadas que - tanto en francés como en español- vienen en imperfecto, se traducirían en polaco mediante una forma imperfectiva, mientras que las demás quedarían en perfectiva.

En el segundo ejemplo tampoco podemos decir que el aspecto se sobreponga a la significación temporal puesto que no es la temporalidad la que entra aquí en juego. Visto desde la perspectiva del momento de habla, la acción es evidentemente pasada. En este caso se trata del hecho que el aspecto imperfectivo representado por el imperfecto se sobrepone al. aspecto perfectivo propio de los conceptos perlectivos de igual modo como en el caso de ROBAR descrito más arriba. De este modo el imperfecto (igual como el presente) puede expresar el aspecto imperfectivo dominante y sirve para dramatizar la acción, alargando el momento en cuestión. Recordemos que en el presente de los perfectivos el mismo papel lo desempeña la perífrasis 'estar + gerundio': Le ha dado un dolor tan fuerte que está muriéndose. Solo falta añadir que en la lengua polaca esta función del imperfecto español sc cxpresa también con las formas verbales imperfectivas.

El tercer punto está un poco más complicado, ya que dos factores lingüísticos entran aquí en el juego. No obstante, estamos totalmente de acuerdo con la explicación del uso del imperfecto de cortesía propuesta por Gili: el papel más importante io desempeña, aquí también, la continuidad propia del imperfecto. Sin embargo este no es el único motivo de su uso en esta función. Comparando el imperfecto de cortesía español con su traducción polaca recibimos lo siguiente:

Quería pedirle un favor se traduce en polaco también con una forma imperfectiva en pasado, pero ya Venía a pedirle un favor se expresa en polaco en PRESENTE, pues también en una forma imperfectiva. Asimismo la forma polaca no 
pierde su valor suavizante ${ }^{8}$. Otras formas de este grupo citadas por Gili y Gaya (1979: \$124) se expresan en polaco en potencial. De todos modos, aquí también se trata de alejar de cualquier manera lo abrupto de la petición haciendo más dificil la negativa del oyente. (Fleichman;1983; 186) ${ }^{9}$. Obviamente, estas conductas estan profundamente arraigadas en nuestras culturas y deben reflejarse en nuestro lenguaje. Afirmamos que, desde el punto de vista lingüístico, el principal candidato a este papel es —además del modo potencial - el imperfecto de cortesía. Por cjemplo, Fleichman afirma que la forma ideal para cumplir esta función en inglés es el pasado y $\mathrm{M}^{\mathrm{a}}$ Luz Gutiérrez Araus - en su Relevancia del discurso en el uso del imperfecto- que en español es el imperfecto el que desempeña ese papel, porque el español no tiene como el inglés una forma simple del pasado sino dos ${ }^{10}$. Sin embargo, la explicación puramente sintáctica no nos parece bastante para explicar las razones semánticas y/o extralingüísticas de este uso. La clave para entender la coherencia de nuestros actos comunicativos es la comprensión de las reglas semántico-sintácticas junto a una serie de factores extralingüísticos y sus correlaciones entre sí. ¿. Cúal es pues el motivo de elegir el imperfecto para esta función? En nuestra opinión dos factores entran aquí en el juego: uno de ellos es el valor de pasado que permite al. hablante distanciarse de lo enunciado. En consecuencia se elije el imperfecto por su carácter imperfectivo, que le hace apto para expresar también una posibilidad indeterminada. Es menester recordar que en polaco es este carácter imperfectivo, la continuidad, el único signo lingüístico de la sumisión del hablante a la voluntad del interlocutor en el caso de Venía a pedirle perdón. (Przychodze prosić o przebaczenie). Nótense también que al. sustituir el imperfecto por el potencial resaltamos también la distancia de lo enunciado por el carácter irreal del modo, pero el valor imperfectivo no se pierde.

\footnotetext{
8 'Enunciamos modestamente nuestra pregunta o nuestro deseo en imperfecto, como algo iniciado cuya consumación o perlección hacemos depender de la voluntad de la persona a quien nos dirigimos'(Gili y Gaya, \$161).

"Citido por M Luz Gutićricz Araus en su Relevancia del discurso en el uso del imperfecto, cn: Revista Española de Linguística,26,2, págs. 327-336.

1" (Ibil.., 332). Nos parece importante aludir a este interesante artículo de $M^{\mathrm{a}}$ Luz Gutiérez Araus ya que cn este artículo la autora aborda el mismo problema de usos secundarios del imperfecto aunque su supuesto es diferente: según $\mathrm{M}^{\mathrm{a}}$ Luz Gutiérrez Araus los diversos valores del imperfecto no tienen ninguna relación con el aspecto, ya que 'es el <presente del pasado> y de ahí que cl contenido del concepto <simulaneidad> se haya confundido con el de <aspecto verbal durativo>'. Sin embargo, nos parece muy acertado su otro supuesto,es decir, que todos los valores secundarios del imperfecto - tanto los discursivos como los pragmáticos- están relacionados con estrategías del hablante. Sin cmbargo hasta ahora sólo se ha podido listar o, por lo menos clasificar los valores secundarios de las formas gramaticales como lo hace, por ejemplo, con gran precisión $\mathrm{M}^{\mathrm{a}}$ Luz Gutiérrez Araus. Ahora, por fin, la gramática cognitiva, gracias a su estrategía investigadora, permite penetrar en este campo hasta ahora poco explorado por falta de medios adecuados.
} 
Y para terminar, los casos 4 y 5 parecen ser muy similares al. caso $n^{\circ} 3$ (aunque el $5^{\circ}$ punto nos parece un caso especial del punto $4^{\circ}$, es decir una condicional sin prólasis: si fuera posible, yo sería / era una princesa y tú...). Así pues, la posibilidad de emplear el imperfecto por el potencial parece ser, también aquí, la continuidad, el valor imperfectivo del primero más cl carácter 'distancial' del pasado.

Como acabamos de ver el carácter continuativo (imperfectivo) del imperfecto, que es su función primaria, sienta - también aquí - la base de todas las funciones periléricas desempeñadas por esta forma gramatical. Recordemos también que a la iuz de los ejemplos aquí analizados la función primaria, protolípica de las proposiciones expresadas en presente gramatical es denotar las situaciones actuales, es decir simultáneas a la situación de la enunciación. Además todos los usos periféricos del presente gramatical responden a una misma característica gramatical como base de su convencionalización: la continuidad (imperfectividad).

En conclusión, aunque en nuestro estudio nos hemos contentado con:

1. separar la función prototípica de las funciones periféricas de dos tiempos gramaticales: presente e imperfecto;

2. exponer y corroborar el hecho de que una función prototípica es lingüísticamente autónoma;

3. exponer y corroborar que las propiedades puramente lingüísticas (nocionales) -como en nuestro caso la imperfectividad - deben jugar un papel central en la creación de los cuadros convencionalizados ('schematized interactive frames') que condicionan los usos secundarios de las formas gramaticales.

Por todo lo anterior nos parece haber puesto claramente de manifiesto que, aunque a la luz de la lingǘstica cognitiva, es obvio que la frontera entre el conocimiento linguístico y conocimiento del mundo es artificial, las reglas scmánticas juegan un papel básico en (por lo menos) ciertos fenómenos cognitivos.

\section{Referencias bibliográficas:}

Alcina, J. \& Blecua, J. M. (1975): Gramática española, Barcelona: Ariel. Banys, W., (1997): 'Temps, Modes \& Relations Temporelles dans les propositions introduites par <si>: si 'standard', en K.Bogacki \& T.Zielińska-Giermak (eds.)

Espace \& Temps dans les Langues Romanes et Slaves, Actes du Colloque, 19-

21 wrzesień 1996, Varsovia, Instytut Filologii Romańskiej, págs.34-52.

Bello, A., (1970 ed.): Gramática de la lengua castellana, Buenos Aires: Sopena. Bosquc, I., et al. (ed.) (1990): Tiempo y aspecto en español, Madrid: Cátedra. 
Bogacki, K. \& Karolak, St. (1991): «Fondements d`une grammaire à base sémantique», Lingua e Stile,.XXVI-3, págs.309-345.

Cifuentes Honrubia J. L. (ed.) (1998) Estudios de lingüística cognitiva, I \& II, Alicante: Universidad de Alicante.

Cuenca M. J. \& Hilferty J. (1999): Introducción a la lingüística cognitiva, Barcelona: Ariel.

Fernández Pérez, M. (1993): «Sobre la distinción aspecto vs. Aktionsart», ELUA, 9, págs. 265-293.

Gili y Gaya, S. (1980): Curso superior de sintaxis española, La Habana: Editorial Pueblo y Educación.

Gutiérrez Araus, M. L. (1997): «Relevancia del discurso en el uso del imperfecto», Revista Española de Lingülística,26,2, págs. 327-336.

Inchaurralde Besga, C. (1998): «La interacción tiempo-modo-aspecto en el verbo.

Una perspectiva cognitiva...», en J. L. Cifuentes (ed.): Estudios de Linguística cognitiva, Alicante: Universidad de Alicante, págs.639-648.

Karolak St. (1986): Kwantyfikacja a temporalizacja (Cuantificación y tcmporalización), en: Studia gramatyczne butgarsko-polskie, I, Temporalność, 65-81, Wroclaw-Warszawa-Kraków-Gdańsk- Łódź: Zak1 ad Narodowy im. Ossolińskich, Wyd. PAN.

Karolak, St. (1997): «Le Temps et Le Modèle de H. Reichenbach», en: Études cognitives, 2 SOW, Varsovia.

Lakoff, G. \& Johnson, M. (1986): Metáforas en la vida cotidiana, Madrid: Cátedra. Langacker, R.W. (1995): «Raising and Transparency», Language, 71, págs. 1-62. Langacker, R.W. (1998): «Indeterminacy in semantics and grammar», en J. L. Cifuentes (ed.): Estudios de Linguística cognitiva, I, Alicante: Universidad de Alicante, págs.

Nowakowska-Kempna, I. (1995): Konceptualizacja uczuć $w$ języku polskim, (Conceptualización de sentimientos en la lengua polaca) WSP, W-wa;

Reichenbach H. (1966): Elements of symbolic logic, Nueva York-Londres: CollierMacmillan Limited.

Rojo, G. (1976): «La temporalidad en español», Verba, 3, págs. 65-89.

Vendler, Z. (1967): «Verbs ands Times», en: Linguistics in Philosophy, IthacaNueva York, págs.97-121.

Wierzbicka A. (1972): Senantic Primitives, Frankfurt: Atheneum.

Wierzbicka, A., (1999): Jezyk - Umyst-Kultura, PWN,W-wa.

Wilk -Racięska, J. (1995): El artículo y la genericidad a la castellana. La distribución en los sintagmas nominales simples, Wydawnictwo Uniwersytetu Śląskiego, Katowice. 
Wilk -Racięska, J. (1997): «Significación de las palabras: ¿el sistema o la convencionalidad?», en: Actas del I Congreso Internacional de Semántica, vol. II, La Laguna: Universidad de la Laguna, (en prensa).

Wilk -Racięska, J. (1998): «La doble vida del adjetivo», en: Neophilologica,13, red. Wicsław Banyś, Wydawnictwo Uniwersytetu Śląskiego, Katowice.

Wilk -Racięska, J. (1998): «How to be a Hypocrite, or Axiology of Diminutives», cn J. L. Cifuentes (ed.): Estudios de Linguística cognitiva, II, Alicante: Universidad de Alicante, págs. 921-930. 